\title{
QUASI-INVERTIBILITY IN A STAIRCASE DIAGRAM
}

\author{
WALTER NOLL
}

We deal with objects and morphisms in an abelian category, e.g., with modules and module-homomorphisms. Any morphism $\alpha: A \rightarrow B$ has a standard factorization $\alpha=\alpha_{m} \alpha_{e}$, where $\alpha_{m}$ is a monomorphism and $\alpha_{e}$ an epimorphism.

Definition. A morphism $\alpha: A \rightarrow B$ is said to be quasi-invertible if it satisfies any one of the following equivalent conditions ${ }^{2}$

(i) There is a morphism $\alpha^{\prime}: B \rightarrow A$ such that $\alpha \alpha^{\prime} \alpha=\alpha$.

(ii) There is a morphism $\bar{\alpha}: B \rightarrow A$ such that

$$
\alpha \bar{\alpha} \alpha=\alpha \quad \text { and } \quad \bar{\alpha} \alpha \bar{\alpha}=\bar{\alpha} .
$$

(iii) $\alpha_{m}$ has a left inverse, and $\alpha_{e}$ has a right inverse.

(iv) $\operatorname{ker} \alpha$ has a left inverse, and coker $\alpha$ has a right inverse.

If $\bar{\alpha}$ satisfies (1) we call it a quasi-inverse of $\alpha$.

For module-homomorphisms, quasi-invertibility means that both Kernel and Image are direct summands.

For monomorphisms, quasi-inverses coincide with left inverses, i.e., monomorphisms are quasi-invertible if and only if they are leftinvertible (or "coretractions"). For epimorphisms, quasi-inverses coincide with right inverses, i.e., epimorphisms are quasi-invertible if and only if they are right-invertible (or "retractions").

The purpose of this note is to state and prove the following result, which was needed in an investigation of annihilators of differential operators [2], but may have other applications.

Theorem. Consider the "staircase" diagram in which dots denote unnamed objects. Assume that the diagram is commutative, that all rows and columns are exact, that the morphisms indicated by double arrows are quasi-invertible, and that either $\xi$ is an epimorphism or $\eta$ a monomorphism. Conclusion: if $\lambda$ is a monomorphism then $\mu$ is a quasi-invertible, and if $\mu$ is an epimorphism then $\lambda$ is quasi-invertible.

Received by the editors February 4, 1969.

1 The research leading to this paper was supported by the Office of Naval Research under Contract NONR 760(30).

2 Cf. [1, p. 264, Proposition 5.1], where, instead of "quasi-invertible," the term "allowable" is used with a somewhat more general meaning. 
(2)

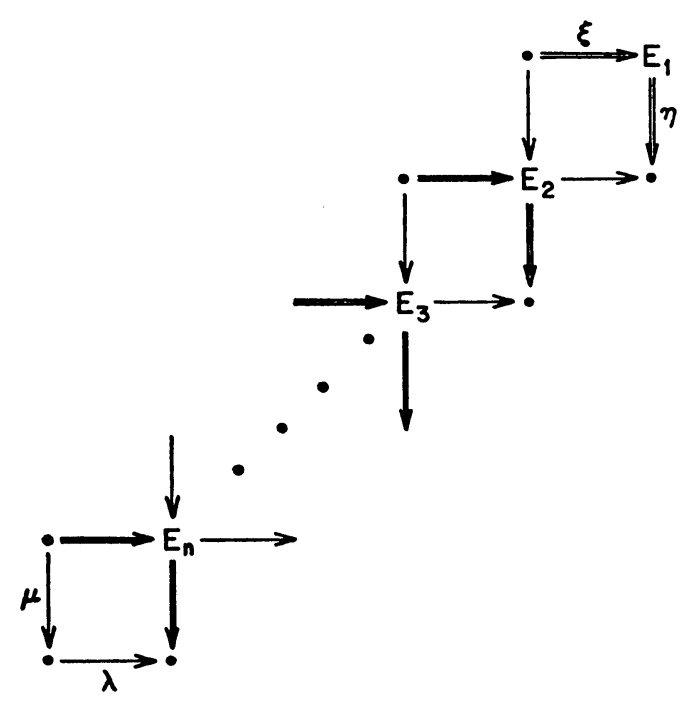

The following easily proved facts will be needed

Proposition A. If $\alpha \beta$ is quasi-invertible and $\beta$ epimorphic, then $\alpha$ is quasi-invertible.

Proposition B. If $\alpha \beta$ is quasi-invertible and $\alpha$ monomorphic, then $\beta$ is quasi-invertible.

If $\alpha$ and $\beta$ are quasi-invertible, we cannot conclude that $\beta \alpha$ is also quasi-invertible. However, the following lemma allows us to draw this conclusion under an additional condition.

Lemma. Consider the "cross" diagram:

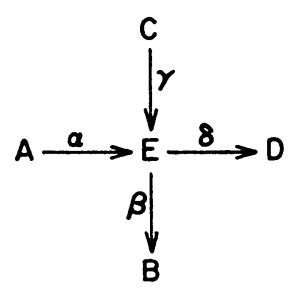

Assume that row and column are exact, and that $\alpha, \beta$ and $\delta \gamma$ are quasiinvertible. Then $\beta \alpha$ is also quasi-invertible.

Proof. Consider the standard factorizations $\gamma=\gamma_{m} \gamma_{e}$ and $\delta=\delta_{m} \delta_{e}$. Since $\delta \gamma=\left(\delta_{m} \delta_{e} \gamma_{m}\right) \gamma_{e}$ is quasi-invertible, it follows by Proposition A 
that $\delta_{m} \delta_{e} \gamma_{m}=\delta_{m}\left(\delta_{e} \gamma_{m}\right)$ is also quasi-invertible. By Proposition B we can conclude that $\delta_{e} \gamma_{m}$ is quasi-invertible. Noting that $\operatorname{Im} \gamma=\operatorname{Im} \gamma_{m}$ and $\operatorname{Ker} \delta=\operatorname{Ker} \delta_{e}$, we see that there is no loss of generality if we assume that $\delta$ is an epimorphism and $\gamma$ a monomorphism. In view of the exactness of the row and the column of the diagram, we may actually assume that

$$
\gamma=\operatorname{ker} \beta, \quad \delta=\operatorname{coker} \alpha .
$$

Now let $\bar{\alpha}$ be a quasi-inverse of $\alpha$, so that

$$
\left(1_{E}-\alpha \bar{\alpha}\right) \alpha=\alpha-\alpha \bar{\alpha} \alpha=0 .
$$

It follows that $1_{E}-\alpha \bar{\alpha}$ annihilates $\alpha$ and hence must factor through coker $\alpha=\delta$. Thus, the exactness of the row of the diagram (3) is expressed by

$$
\delta \alpha=0, \quad 1_{E}-\alpha \bar{\alpha}=\bar{\delta} \delta,
$$

where $\bar{\delta}: D \rightarrow E$. Similarly, one can prove that the exactness of the column of the diagram (3) is expressed by

$$
\beta \gamma=0, \quad 1_{E}-\bar{\beta} \beta=\gamma \bar{\gamma},
$$

where $\bar{\beta}$ is a quasi-inverse of $\beta$ and $\bar{\gamma}: E \rightarrow C$. Incidentally, it is easily seen that $\bar{\delta}$ is a right inverse of $\delta$ and that $\bar{\gamma}$ is a left inverse of $\gamma$, but this fact will not be needed.

Let $\Phi$ be a quasi-inverse of $\delta \gamma$, so that

$$
\delta \gamma \bar{\phi} \delta \gamma=\delta \gamma,
$$

and put

$$
\bar{\psi}=\bar{\alpha}\left(1_{E}-\gamma \bar{\phi} \delta\right) \bar{\beta} .
$$

By (4) and (5) we then obtain

$$
\begin{aligned}
\beta \alpha \bar{\psi} \beta \alpha & =\beta \alpha \bar{\alpha}\left(1_{E}-\gamma \bar{\phi} \delta\right) \bar{\beta} \beta \alpha=\beta\left(1_{E}-\bar{\delta} \delta\right)\left(1_{E}-\gamma \bar{\phi} \delta\right)\left(1_{E}-\gamma \bar{\gamma}\right) \alpha \\
& =\beta\left(1_{E}+\bar{\delta} \delta \gamma \bar{\gamma}-\bar{\delta} \delta \gamma \bar{\phi} \delta \gamma \bar{\gamma}\right) \alpha .
\end{aligned}
$$

It follows from (6) that the last two terms cancel and hence that $(\beta \alpha) \bar{\psi}(\beta \alpha)=\beta \alpha$. Therefore, $\beta \alpha$ is quasi-invertible. Actually, $\Psi$ is easily shown to satisfy also $\Psi(\beta \alpha) \bar{\psi}=\bar{\psi}$ and hence is a quasi-inverse of $\beta \alpha$. Q.E.D.

Proof of Theorem. Suppose that $\xi$ is an epimorphism. The upper right corner of the staircase diagram (2) can then be used for the construction of the following cross diagram 


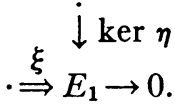

$$
\begin{aligned}
& \Downarrow \eta
\end{aligned}
$$

It is clear that the hypotheses of the lemma are satisfied, and it follows that $\eta \xi$ is quasi-invertible. The same conclusion is obtained in a similar manner if $\eta$ is a monomorphism.

Using the commutativity of the diagram (2), we see that the hypotheses of the lemma are satisfied for the cross diagram centered at $E_{2}$. Proceeding by induction, we conclude that the lemma can be applied to the cross diagram centered at $E_{n}$ and hence that $\lambda \mu$ is quasi-invertible. The assertion follows now by applying Proposition B or Proposition A. Q.E.D.

\section{REFERENCES}

1. S. MacLane, Homology, Springer-Verlag, Berlin, 1963.

2. H. D. Dombrowski and W. Noll, Annihilators of differential operators (to appear).

Carnegie-Mellon University 